\title{
DESIGN AND DEVELOPMENT OF A NOVEL TESTING RIG FOR THE EXAMINATION OF ADDITIVELY MANUFACTURED AUXETIC COMPONENTS
}

\author{
Urquhart, Lewis (1); \\ Fingland, Craig (1); \\ Wodehouse, Andrew (1); \\ Loudon, Brian (2) \\ 1: University of Strathclyde; \\ 2: Loud1Design
}

\begin{abstract}
This paper reports upon the design and development of a novel testing rig for the examination of additively manufactured auxetic componentry. By firstly reviewing the key challenges for practical researchers and exploring the range of approaches used to examine auxetic structures, we subsequently introduce a new testing configuration seeking to enhance the existing methods found within the literature. The developed testing configuration includes a novel mechanical design with a new method for component mounting offering advanced control of the boundary condition and a fully developed control interface which facilitates real-time analytics, a range of data acquisitions and integration with a CAD environment. This paper describes both the development of the mechanical design and the development of the control interface by exploring the key design features and documentation of the manufacturing and assembly process. Finally, we discuss how the presented testing configuration offers a new and flexible way of testing auxetic componentry with additional insights offered for future researchers who wish to recreate or adapt the testing setup for their own examinations of additively manufactured componentry.
\end{abstract}

Keywords: Auxetics, Mechanical analysis, Additive Manufacturing, 3D printing, Evaluation

\section{Contact:}

Urquhart, Lewis William Robert

University of Strathclyde

Design Manufacturing and Engineering Management

United Kingdom

lewis.urquhart@strath.ac.uk 


\section{INTRODUCTION}

Researchers focused on the study of additively manufactured components face a variety of issues in how best to setup test parts and what properties to examine. A wide variety of different strategies have been explored to examine properties like stress, strain and mechanical fatigue. While lots of testing setups for exploring the mechanical behaviour of small to medium sized components have been presented within the research literature, there lacks a consistent approach. Some testing strategies focus on optical analyses, others use sensors and measurement gauges to establish dimensional changes to components. This paper presents the design and development of a novel testing rig expressly built for the examination of 3D printed auxetic components. By initially considering the approach other researchers have applied to the problems of testing physical samples, notably the control of the boundary conditions, we present the development of a new system that integrates aspects of a range of analysis tools and offers a novel approach for dealing with the boundary problem. The paper is largely structured to explore the particular challenges posed by the mechanical investigation of flexible structures and demonstrates how the new testing system is configured and built. This is split into three key sections. Firstly, the novel design developed by the authors is introduced where the key mechanical and control design innovations are laid out. This is split into description of the conceptualisation and development process followed by descriptions of the mechanical design and the control interface design. Secondly, we will discuss how the novel integrated system can provide the user a great range of control over the testing procedures and data outputs and thirdly we offer insights for future researchers wishing to recreate or adapt our novel testing setup.

\section{AUXETICS: CONSIDERING CURRENT PHYSICAL TESTING STRATEGIES}

\subsection{Key design challenges in testing auxetics}

An auxetic material is defined by its negative Poisson's ratio leading to interesting mechanical behaviours, notably the material structure will stretch perpendicular to an applied stress. Hou and Silberschmidt (2015) state that there are three basic structures that exhibit NPR properties: re-entrant structures, chiral structures and rotating rigid structures. These structures are created most commonly using additive manufacturing processes and include flexible structures with embedded compliant mechanisms usually produced from polymers and static structures usually produced from metals (see Kolken and Zadpoor, 2017). Auxetic structures have presented several key problems within practical research due to their structural complexities. As simulation offers "idealised" conditions in which to examine components of different material makeups, physical testing in reality leads often to many issues such as control of the boundary conditions and which parameters should be prioritised for measurement in a testing setup. Many practical approaches explored by recent research utilise methods of optical analysis where the parts are assessed by comparison and measurement of relative positions of features. Other more technical approaches have utilised real-time measurement systems to analyse force and deformation dynamics. From a review of the literature focused on the physical testing of auxetics, we can identify a number of distinct treatments of the boundary condition problem i.e. how the parts are held as they are being deformed by tension or compression forces associated with several distinct jig configurations.

Perhaps the most conventional means of administering the mechanical forces to the part is compression between two plates. This is seen in a very simple setup by Abdel-Rahman and Tafrihi (2018) who use rudimentary plastic cuboidal structures to constrain the parts as they are compressed and in an advanced setup by Jiang and $\mathrm{Hu}$ (2017) using a low-velocity hydraulic press. In both instances, the boundary problem is addressed the same way - the part is not held but positioned correctly as force is applied. While the later employs advanced sensor technology, the former derives results from a simple optical analysis.

Another set of methods have used vices, holding the component at the bottom and top as it is compressed or pulled apart. Research from Zulifqar, Hua and $\mathrm{Hu}$ (2018) for example used a fixed jawvice and a movable jaw-vice in conjunction with a high-speed camera for a kind of optical analysis. Markers are placed directly on the part to track the relative position of the points as the part in subjected to tension. The change in the relative position of the points is then interpreted by a computer, providing an evaluation of how the part behaves. A similar approach was taken by 
Dubrovski and others (2019) who examined the behaviour of very thin auxetics. The work used time lapsed photos to record the changes to the structure - a practical method with few complications but less rigorous. Also, Tanaka and others (2017) utilise an especially designed jig with the same analysis method.

Perhaps the most interesting means of securing the components found in the literature involves securing the part with an added support structure essentially moving the point of boundary away from the area being examined. Brighenti and others (2016) use an optical analysis approach to study a twodimensional polymer auxetic. A structure has been added to either side of the part that integrates into a jaw type vice meaning the edge of the area of focus is not grasped directly. This is also seen is metal auxetic testing in work from Alomarah and others (2017).

Lastly, we identified an interesting mounting configuration developed by Auricchio and others (2019) where the samples were secured with the use of a set of supporting truss structures. The pins are placed uniformly within the auxetic structure itself. This means that the boundary condition can be more carefully controlled, and the setup potentially facilitates more complex local deformation tests. The setup is also somewhat flexible, allowing constrains to be applied to some areas and not others. The researchers used an optical analysis method to determine the results. With respect to these approaches, we reviewed how they could be used in the development of a novel setup and the next sections will present the evolution of the design.

\section{DESIGN AND DEVELOPMENT OF A NOVEL TESTING SETUP}

The final rig configuration and testing setup that was developed is shown below in Figure 1. The following sections will detail the development of this novel configuration, splitting the description down into the novel mechanical design features and the design of the control interface.

\section{Mechanical design features:}

- Combined tensile and compressive testing: The new testing rig permits both type of testing without the need for additional components or re-mounting of samples. This reduces time spent during test setup and improves consistency between tests.

- Non -Orthogonal force application: The new adjustable sample mount allows the force application angle to be altered which allows a diverse range of tests to be carried out.

\section{Control interface features:}

- Geometry scanning: A Rhino-Grasshopper definition converts snapshots of each sample into CAD geometry which can be quickly and accurately dimensioned in post processing. This saved geometry creates a physical log of each test which can be used for future reference.

- Automatic Data Management: Grasshopper integrates with any computer to create folders for organising test files. Furthermore, test data is automatically sent to the specific worksheet which relates to that particular test.

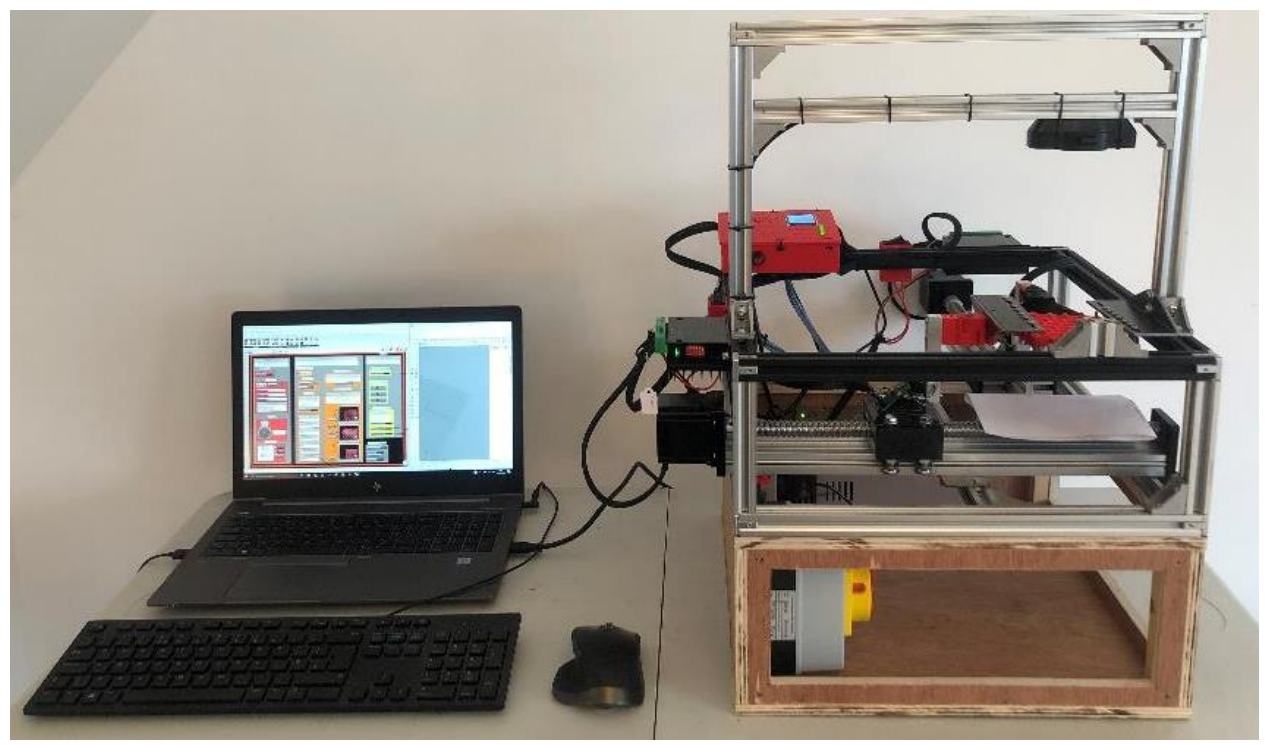

Figure 1: Control interface with fully assembled testing rig 


\subsection{Mechanical design: sample mounting and force application}

Following the creation of a product design specification which identified adaptability in terms of part mounting, real time deformation analytics and non-orthogonal loading as the core requirements of the testing rig, rough sketches were produced to explore different ideas for testing small auxetic sample (Fig 2). The testing rig was centred around mounting and deforming standard $70 \times 70 \times 10 \mathrm{~mm}$ auxetic samples. It was noted that there were two primary elements that the rig design must address. The first of which involved the mounting of a sample in the rig while permitting the freedom to expand and contract in an auxetic manner. It became apparent that this ' $\mathrm{C}$ shaped' mounting system would have interference issues when conducting a compression test. Therefore, the concept was developed further using an upper and lower 'L shaped' design to counteract this issue. The new mount design attached the sample courtesy of a series of pins trapped on a rail inspired by the configuration presented by Auricchio and others (2019). The y axis mount featured the traditional L shape orientation, while the $x$ was rotated though $180^{\circ}$.
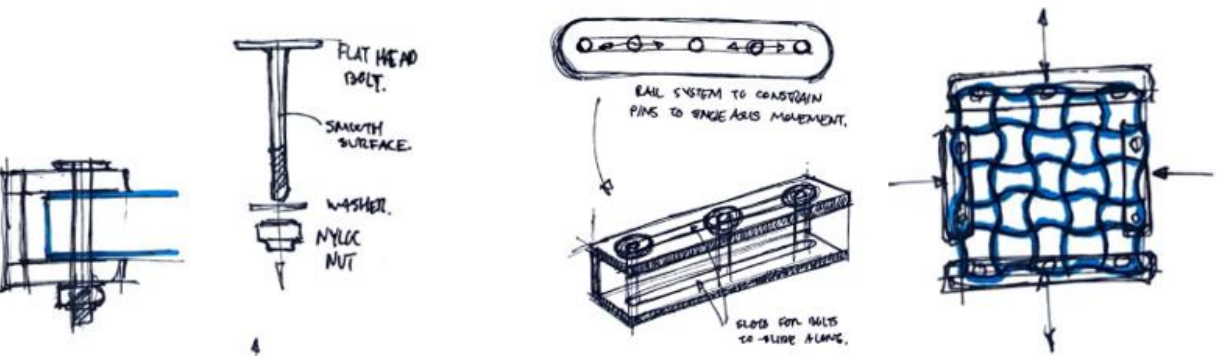

Figure 2: Initial mounting bracket concepts

The second crucial aspect of the design was the means of force application. The specification demanded a system which was capable of both tensile and compressive testing. Two force application concepts arose during concept generation. Initial force concept A (Fig 3, left) involved a modified lathe chuck which offered even force application on all sides. Initial force concept B (Fig 3, right) featured two rails which were each constrained to a single axis. The position of these rails on their corresponding axis (and force applied to sample) was determined by a rotating threaded bar. Furthermore, the sample is contained on both axis in the bottom left corner which creates a form of stationary point.

At this stage, both concepts were viable ideas and each warranted further exploration and development. To assess feasibility and effectiveness, an early prototype was created for each design. Concept B was eventually favoured after assessment due to the ability to carry out displacement testing across larger distances. Concept A was limited by the size of the chuck faceplate whereas concept B could facilitate the use of long threaded bars, allowing much more flexibility in terms of the amount of tensile force that could be applied. Following the selection of this design, a detailed CAD model was produced which the next section will elucidate.
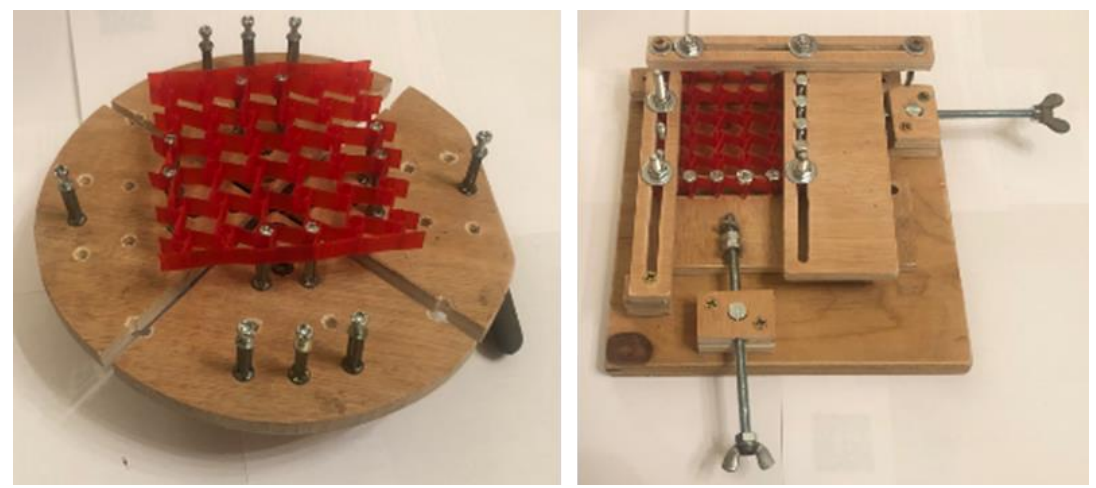

Figure 3: Force concept $A$ (left) and concept B (right) prototypes

\subsubsection{CAD modelling}

A schematic plan was generated to roughly layout the rig elements before creating the final model in SolidWorks (Fig 4). This was also crucial in selecting the various additional components that would be required for the realisation of an effective rig design. 

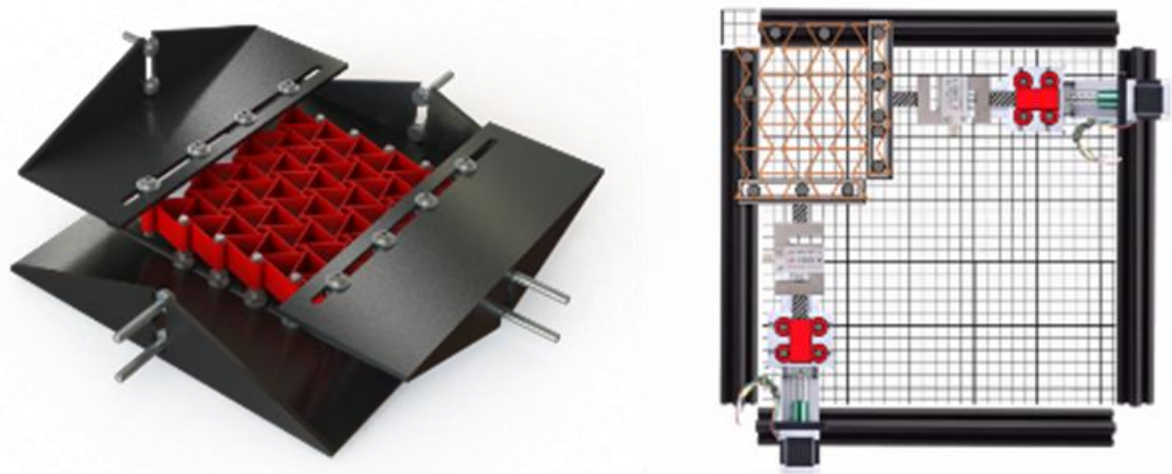

Figure 4: Sample mounting CAD design and test bed schematic showing position of motors

As the CAD model became more detailed, new components such as a camera were added to further improve the functionality of the rig. This would allow snapshots to be taken during each test for use as future reference. At this point, the camera model was simply symbolic of the snapshot function and would later become far more streamlined. Several issues were predicted at this stage which needed to be resolved in the next iteration. The first of which was a lack of access underneath the rig to adjust position of the linear slides. This flexibility when setting up the rig is crucial to permit non-orthogonal force testing. The second problem is the difficulty in mounting samples as there is no quick release mechanism. Another feature requirement that emerged was angular loading of the samples. Tensile and compressive tests are typically carried out at perfect orthogonal angles to the test sample in question. However, this only exposes the auxetic structure to a perfectly uniform forces which are unlike most potential use-cases. Therefore, the testing rig was designed feature non-orthogonal testing in addition to the traditional methods. An adjustable bracket was designed in SolidWorks to permit adjustments to angle of force application. The fundamental angles to be tested were $30^{\circ}, 45^{\circ} \& 60^{\circ}$, so a system was devised to allow adjustments in $15^{\circ}$ increments using a series of teeth. Figure 5 (left) is the Solidworks model which was used to virtually test the functionality of the concept before 3D printing. The parts were then $3 \mathrm{D}$ printed in ABS with $100 \%$ infill to ensure the mount is as strong as possible. This is crucial as any deformation in the mount would result in inaccurate force and displacement readings. This solution to non-orthogonal force application is functional in tests involving low magnitudes of force. In the event of increasing the load to test samples to failure, the mount should be removed or recreated using metal. As can be seen in Figure 4, the previous issues were addressed in the final model. A wooden framework was added to allow access beneath the rig as well as a sliding quick release for attaching/detaching samples. As the testing process was considered further, several other small additions were made to enhance the procedure and further improve the testing rig. This included the addition of a user interface, several power switches and stepper motor drivers.
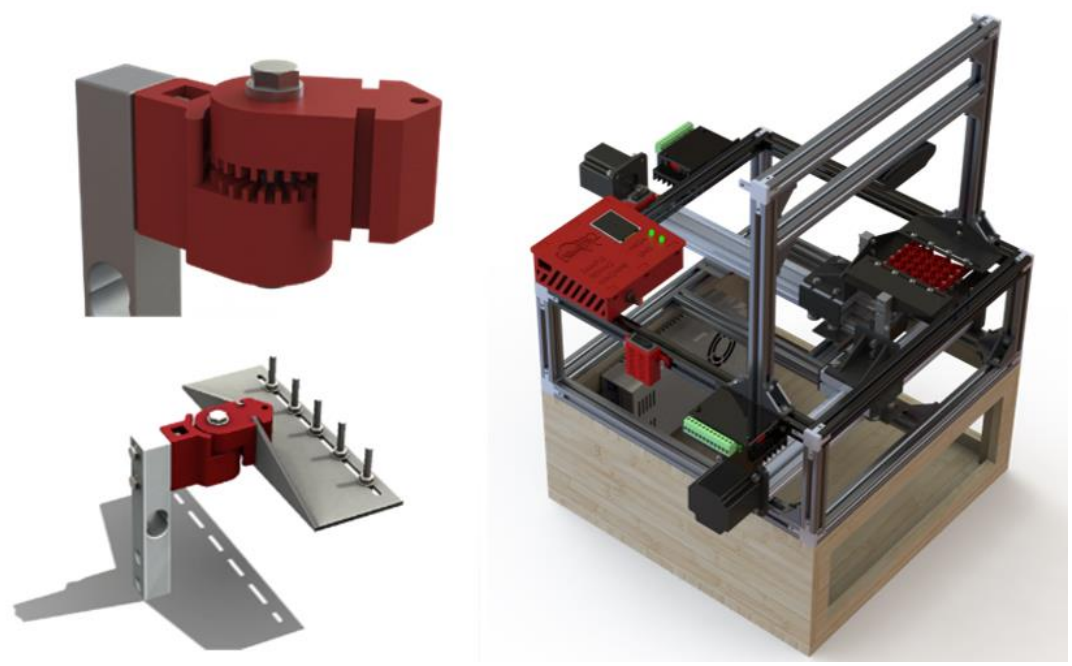

Figure 5: Adjustable sample mount facilitates non-orthogonal loading (left) and final CAD assembly (right) 


\subsubsection{Rig manufacture}

To create the testing rig, a combination of manufacturing techniques were employed (see Figure 6). The rig features a structural framework made exclusively from 2020 extruded aluminium. This is very adaptable and permits a wide range of additional attachments while maintaining a high level of strength for heavy duty testing. Beneath the metal frame, a plinth was needed to elevate the rig and provide additional mounting area for components such as the power supply and isolation switch. This was fabricated from plywood using a table saw and jig saw before being connected together with woodscrews. Once the wooden plinth was manufactured, it was bolted to the framework above to secure it in place. 3D printing was utilised to create bespoke housings for the control panel and stepper motor switches which would have otherwise been very time consuming to manufacture.
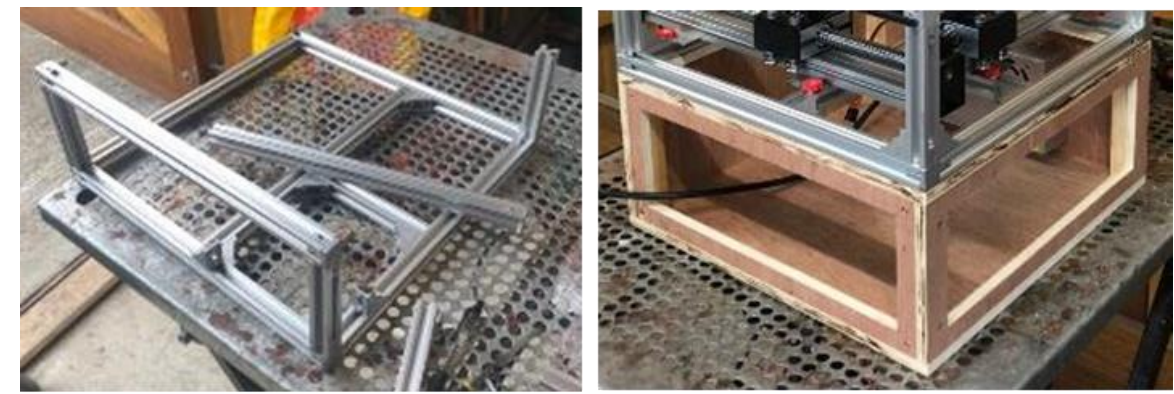

Figure 6: Construction and assembly of testing rig

Following the manufacture of the testing rig framework, the electronics could be integrated into the system, a full schematic of which is shown below in Figure 7. A purpose-built control panel box was designed and printed to house all the complex electronics. When closed, this acts as a control panel to view rig information and adjust certain testing variables such as target length. The box features ventilation holes on the side to encourage airflow and prevent the Arduino chips overheating.

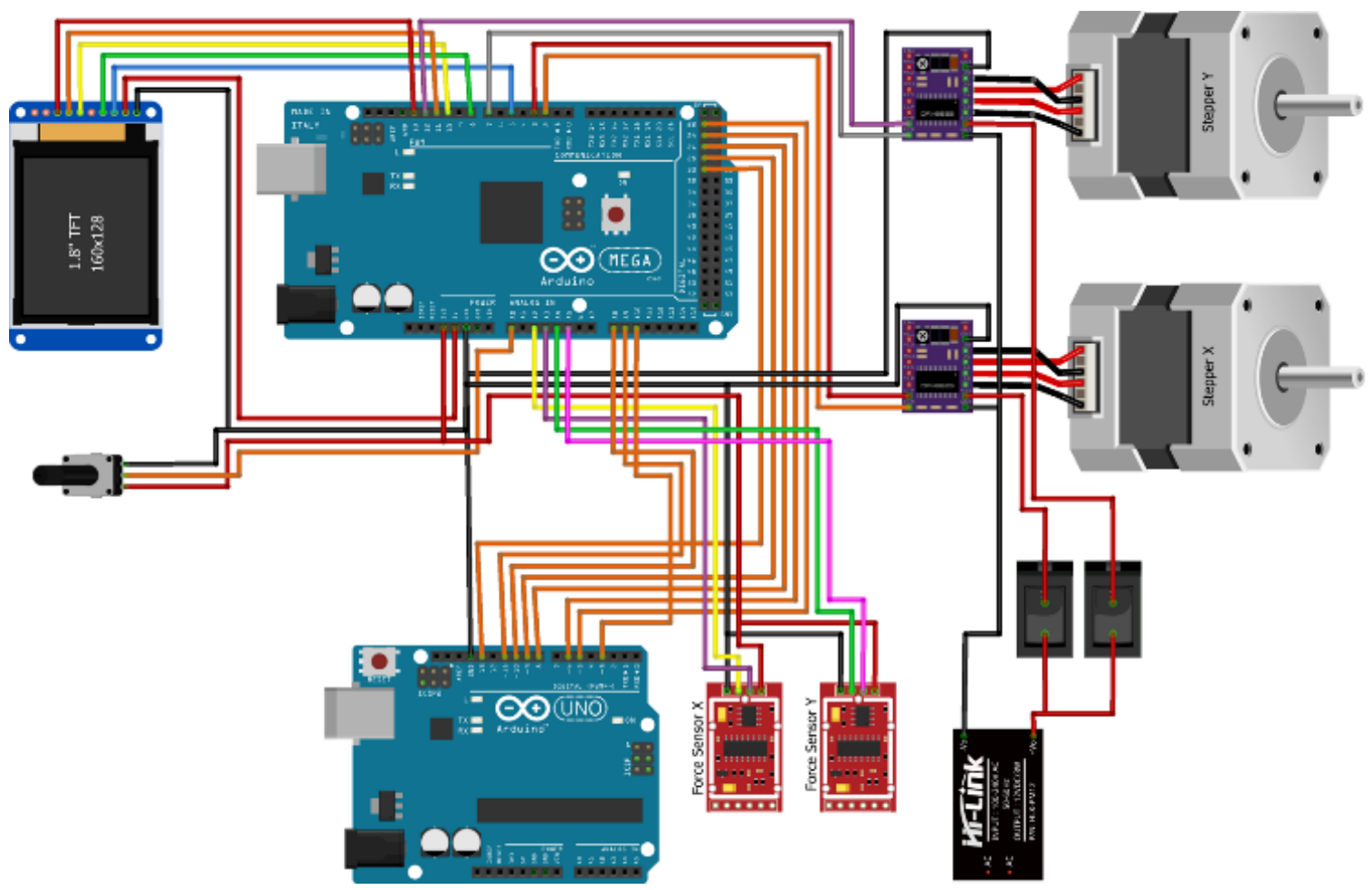

Figure 7: Testing rig electronics schematic.

After completing the various sub elements of the testing rig, the final assembly was carried out. Standalone components such as the webcam and USB extension were attached using cable ties (these were designed to be freely movable therefore were not permanently fixed). Safety features such as the 
isolation switch and stepper off switches were added to protect the user and machine should any problems arise during use.

\subsection{Control interface: design of analysis algorithm}

A Rhino-Grasshopper definition was used to explore the creation of auxetic structures and to control the testing of those structures in the mechanical testing rig developed here. Grasshopper is a visual programming language and environment that runs from within the CAD package Rhinoceros 3D (usually shortened to "Rhino"). The rationale behind selecting Grasshopper was primarily due its versatility. There are very few programs which can facilitate such a vast variety of tasks within a single window. Furthermore, the visual style of programming is faster and easier to get a basic grasp of than traditional coding making it very user friendly. The following sections will detail the creation of the control interface.

\subsubsection{Control panel}

For the benefit of the reader, the "control panel" algorithm developed for the testing will be examined in its distinct elements along with descriptions of the user interface that accompanies this. A Grasshopper definition was created to control the testing rig and combine a number of different process into a single program. This allowed the user conducting the test to monitor, record and export data all from a single window within Rhino. Figure 8 shows the virtual control panel which features controls required for carrying out a full test.

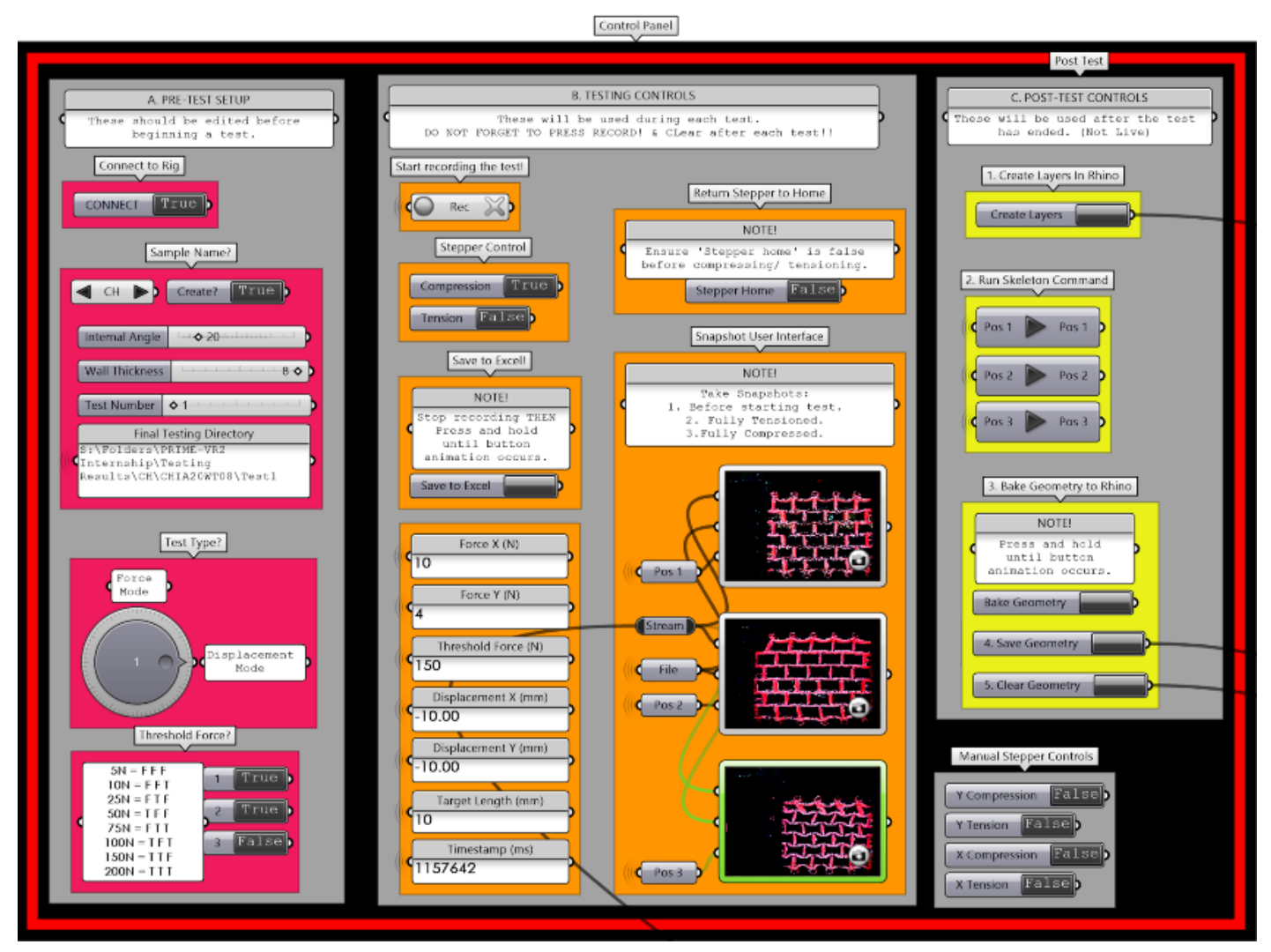

Figure 8: Testing rig virtual control panel

The panel is split into three clear sections which each operate a different stage of the procedure:

A) Pre-test setup: Prior to testing, Grasshopper connects to the rig via the first toggle switch. The test sample is then be named using the series of sliders which automatically creates a folder and file name in an assigned directory. Following this, the type of test is selected from Displacement (deform sample to xMillimetres) or Force (deform sample to xNewtons). Finally, the threshold force is selected from a list of pre-determined values. If the force meters read above threshold force at any point, the rig will stop to prevent damage. 
B) Testing controls: This section of the panel provides control of rig functions during each test. The record component is activated to log the data output from the Arduino until the stop button is pressed. The snapshot components create a visual record of three different positions (typically stationary, under max tension and under max compression. The stepper motors are activated by toggle switches and deform the sample in either the tension or compression direction. The testing rig will continue to deform the sample in one direction until either the target length or threshold force has been reached. Finally, after the desired testing has been competed for one sample, the recording can be stopped, and results exported to Excel via the 'Save to Excel' function. Finally, the steppers are returned to the start position by the 'return to home' switch. This allows the sample to be easily removed in preparation for the next test.

C) Post-test controls: This section permits the transfer of geometry data from Grasshopper into Rhino. The 'create layers' toggle switch inserts six named and coloured layers in the Rhino window (two for each recorded position). These act as a destination for sending geometry later in the process. The three play buttons (data dams) allow for extensive commands to be run once and reduce the risk of the program crashing due to lack of available memory. In the background, Grasshopper will convert the photos of each position into scaled mesh geometry and skeleton traces which can be sent to Rhino. After the 'bake geometry' button is activated, the six geometry files will be sent to Rhino and onto their respective layers. Following this, the Rhino file (including geometry and layers) can be saved using the 'save geometry' button. Finally, the geometry can be cleared from the window in preparation for the following test. While the control panel provides all the required controls and functions, there is a large amount of additional programming and coding which runs in the background.

\subsubsection{Interacting with control algorithm}

There are several other key benefits to using the Grasshopper definition control interface we have developed which can be reviewed presently:

- Real time control: As can be seen in the virtual control panel, the definition offers many useful features permitting user interaction with the program. The variety of different inputs from sliders to buttons to control knobs provides many options. Figure 9 demonstrates how each sample can be automatically named (and placed in a new folder) by operating a series of drag sliders which control other components in the background. To achieve the same control panel in a physical form would be very complex, expensive and very time consuming. Whereas within Grasshopper, it can be created easily and within a fraction of the time. Furthermore, adjustments and additions to the control panel can be made instantaneously without the need for remanufacturing the it in reality.

- Data Acquisition: Through the use of additional grasshopper plugins, data can be obtained from a multitude of sources within our definition. In the testing rig definition, a plugin known as Firefly is utilised to read data from an Arduino which is connected over USB. Furthermore, Firefly also facilitates the inclusion of a video stream from a Webcam. The combination of these simultaneous data inputs leads to a wealth of information arising from each test. In most webcam uses the entire video stream is required for the task in hand. However, in this case, only the sample needs to be recorded and therefore the background creates problems further in the process. Grasshopper provides a method of editing the live stream to allow only the red-channel to remain. As all the samples are red, this is a quick way to record the sample without the additional noise.

- Integration with CAD Environment: Our definition offers unparalleled integration between programming and the 3D environment. By selecting specific data components in a Grasshopper definition, the user can preview data \& geometries in the Rhino window. Furthermore, by selecting the "bake" command, these forms of data are sent permanently and can be saved to the Rhino file. The ability to save scaled 3D models of each test sample is also useful in post-test analysis. These new 3D models can be compared against the original test sample file (used to 3D print the sample). This also ensures that both the rig and 3D printer is maintaining accuracy. Figure 9 demonstrates how Grasshopper takes a snapshot using a webcam and then converts it into scaled geometry in the Rhino window. Moreover, this geometry is stacked in single layers which can be accessed individually or as a group. 


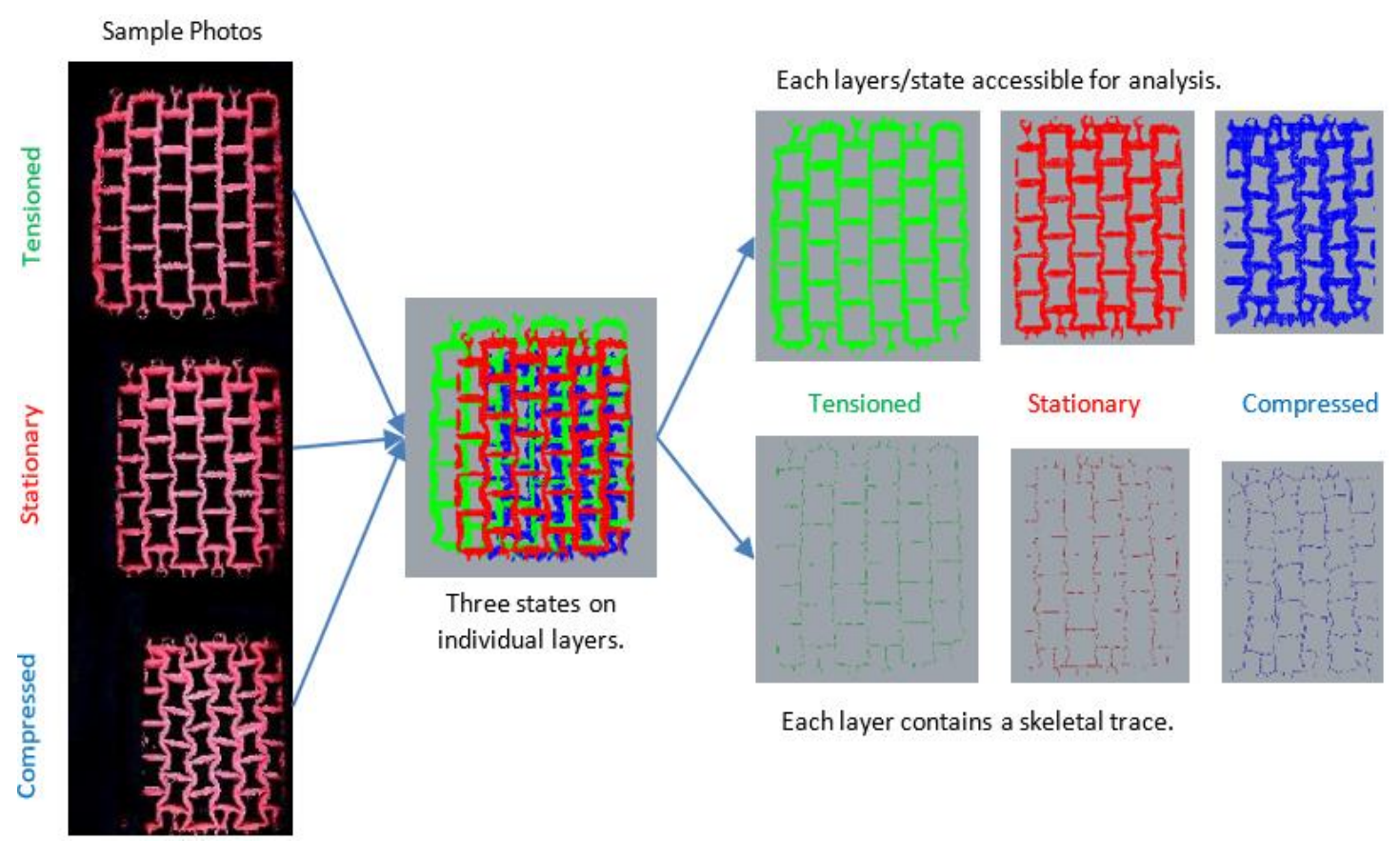

Figure 9: Images of three states are layered to see differences in structures

\subsubsection{Types of data generated}

The first and most important are the measurements relating to forces and displacements. We utilised two force sensing gauges calibrated against the movement of a turning screw controlled by an Ardino motor. This data was stored in real-time on an Excel spreadsheet when it was generated. This was then directly graphed showing the mechanical behaviour across the given testing period. Furthermore, the testing rig was set up to capture the physical states of the parts at rest, at full tension and at full compression. This, combined with the force-displacement data, provides a more complete picture of how the samples respond to the deformation. Figure 10 shows an example of the images that the testing rig can produce. This type of data is useful as it provides a physical analogue for the data points recorded in the Excel sheet. Additionally, it provides a useful comparison for the simulation work where the "behaviour" of the animations can be superficially compared to the actual physical samples.
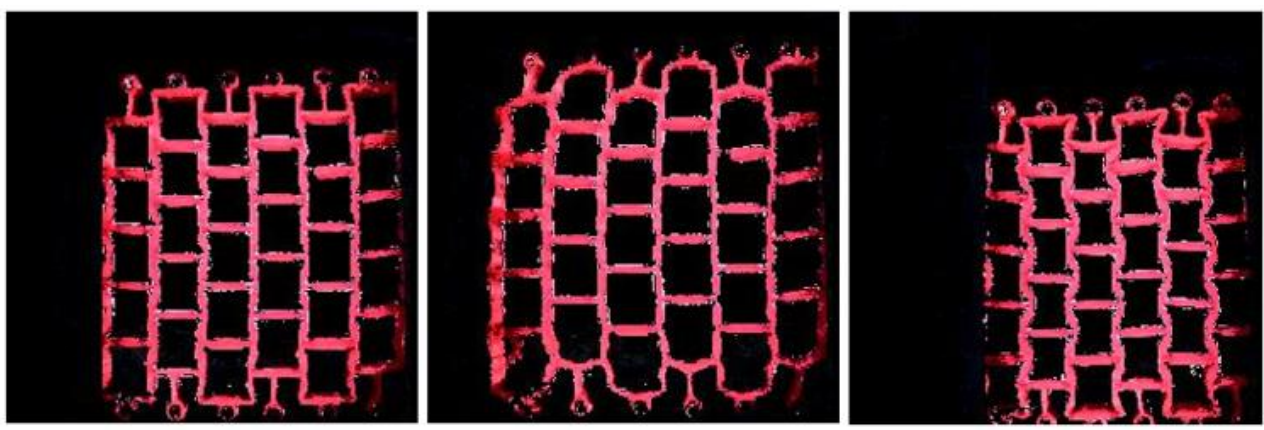

Figure 10: Example of the snapshots generated by the testing rig - at rest (left), at tension (middle), compressed (right)

\section{DISCUSSION}

This previous section detailed the algorithmic work within the Grasshopper environment developed in conjunction with the auxetic sample physical testing rig. The algorithmic definition developed allows for multiple lines of control allowing the careful deployment of tests, the monitoring of the samples during testing and the efficient recording of data. While the system continues to be refined, the control panel affords a great deal of command over the running of the tests as well as real time visualisations of the parts under loading. Ultimately these tests will be used for additional insight into how particular materials and structures behave mechanically - information that can also be fed into the generative 
algorithms for the development of components given a design domain definition. This wholistic approach is something similar to that presented by Yang, Vora and Chang (2018) for example, but has some short comings in terms of sophistication, though does offer a quick and easy method to test multiple parts before moving on to more technical measurement machines. The key benefit is perhaps the control algorithm which appears to be an entirely novel feature in a setup such as this and with no similar such control interfaces identified in the literature. In essence, a setup such as ours could be recreated or adapted by other researchers for the purposes of quick test cycles, facilitating the rapid understanding of mechanical behaviour under particular conditions of selected components.

This setup also offers a novel means of treating the boundary condition problem and applying the forces. In the range of setups explored in the literature, none were identified that facilitated control of all four sides of the part as it was being subjected to mechanical forces. The configuration we present, inspired by that seen in Auricchio and others (2019), using a system of support trusses on all sides creates a more stable system allowing the behaviour to be more carefully controlled during testing. Such a setup will however need validated through some form of mathematical modelling to confirm its accuracy and effectiveness. The setup additionally allows for the testing of non-orthogonal forces acting upon the parts. As most of the literature is focused on the linear, orthogonal application of forces this is a novel feature that may interest other researchers in exploring more dynamic mechanical behaviour.

\section{CONCLUSION}

This work presents the design and development of a novel setup for the testing and real-time examination of auxetic components. The structural design of these components presents a number of challenges for analysis and testing configurations notably the boundary condition problem. By reviewing the work of other practical researchers, several key specifications for the proposed testing setup were developed including adaptability of the mounting system and real time mechanical analytics. The testing rig developed integrates a system of optical analysis with real-time force-displacement data. A novel mounting jig was introduced allowing the part boundary to be controlled at all sides. In addition, a comprehensive control interface was developed in Grasshopper allowing users of the test rig to set up the test in the correct way by providing control of numerous parameters and facilitating the organisation of data. It is anticipated that setups such as the one presented here could be produced or adapted by other researchers interested in auxetics to quickly assess mechanical behaviour of componentry, encouraging more rapid prototyping and more expansive explorations of design space.

\section{REFERENCES}

Abdel-Rahman, A. and Tafrihi, E. (2018). “Heat-Actuate Auxetic Facades”. Conference Proceedings: Facade Tectonics 2018

Alomarah, A. et al. (2017). Mechanical Properties of the 2D Re-entrant Honeycomb Made via Direct Metal Printing. IOP Conf. Ser.: Mater. Sci. Eng. 229012038

Auricchio, F., et al. (2019). "A novel layered topology of auxetic materials based on the tetrachiral honeycomb microstructure". arXiv: Applied Physics.

Brighenti, R., et al. (2016). "Nonlinear deformation behaviour of auxetic cellular materials with re-entrant lattice structure." Fatigue \& Fracture of Engineering Materials \& Structures, 39, 599-610.

Dubrovski, P.D., et al. (2019). "In-Plane Behavior of Auxetic Non-Woven Fabric Based on Rotating Square Unit Geometry under Tensile Load". Polymers, 11.

Hou, X. and V. V. Silberschmidt (2015). "Metamaterials with Negative Poisson's Ratio: A Review of Mechanical Properties and Deformation Mechanisms" in Mechanics of Advanced Materials. NY, USA, Springer: $155-179$.

Jiang, L., \& Hu, H. (2017). "Finite Element Modeling of Multilayer Orthogonal Auxetic Composites under LowVelocity Impact”. Materials (Basel, Switzerland), 10(8), 908. https://doi.org/10.3390/ma10080908

Kolken, H. M. A. and A. A. Zadpoor (2017). “Auxetic mechanical metamaterials.” RSC Advances 7(9): 5111- 5129.

Tanaka, H., Suga, K., Iwata, N., \& Shibutani, Y. (2017). Orthotropic Laminated Open-cell Frameworks Retaining Strong Auxeticity under Large Uniaxial Loading. Scientific Reports, 7.

Yang, C., Vora, H.D., \& Chang, Y. (2017). "Behavior of auxetic structures under compression and impact forces". Smart Materials and Structures, 27, 025012.

Zulifqar, A., Hua, T., \& Hu, H. (2018). "Development of uni-stretch woven fabrics with zero and negative Poisson's ratio". Textile Research Journal, 88, 2076 - 2092. 